\title{
Asymmetric Volatility and Dynamic Asset Allocation
}

\author{
Meng-Sung Hsieh ${ }^{1}$ \\ ${ }^{1}$ Ph.D student of Finance, Feng-Chia University, Taiwan \\ Correspondence: Meng-Sung Hsieh, Ph.D student of Finance, Feng-Chia University, Taiwan
}

Received: April 26, 2016

Accepted: May 11, 2016

Online Published: May 12, 2016

doi:10.5430/afr.v5n2p126

URL: http://dx.doi.org/10.5430/afr.v5n2p126

\begin{abstract}
This paper devises a stochastic volatility feedback (SVF) model to investigate the economic importance of the leverage and volatility feedback effects, both of which are the two main explanations for volatility asymmetry. We perform the dynamic asset allocation model under the SVF model and then assess the economic performances for the corresponding optimal investment strategies. Our findings are as follows. (i) the volatility feedback effect drives the intertemporal hedging demand, in contrast, the leverage effect has a minor effect on it; (ii) a longer investment horizon or a higher current volatility enhance the volatility feedback effect; (iii) ignoring the volatility feedback effect would suffer from tremendous economic loss.
\end{abstract}

Keywords: Leverage effect, Volatility feedback effect, Stochastic volatility, Asset allocation

\section{Introduction}

The empirical evidence to date in the finance literature suggests that there are two major style facts in stock return volatility. First, stock return volatility is time-varying and clustering. The change in volatility is serially correlated and persists for a long period of time. Second, the shocks to stock return volatility are negatively associated with the unexpected stock returns. Typically a large negative shock to stock return may be associated with upward revisions in subsequent return volatility (Note 1). This feature has been called volatility asymmetry. According to the financial theory, it can be explained by the leverage hypothesis and the volatility feedback hypothesis (Note 2). The leverage hypothesis proposed by Black (1976), Christie (1982) and Nelson (1991) advocates that a declined equity value (negative equity return) deteriorates the total value of a leveraged firm and then raises the holding risk for the equity, driving up the equity volatility. The volatility feedback hypothesis suggested by Pindyck (1984), French, Schwert and Stambaugh (1987), Campbell and Hentschel (1992), Bekaert and Wu (2000) and Wu (2001) justified that while the volatility risk is priced (the expected stock returns increase when volatility raises) and the volatility process is clustering and persistent, any shock of either sign increases the future expected volatility and raises the required returns for holding stocks, bringing down the current stock price (negative return).

Leverage and volatility are two interrelated topics which are extremely important for financial managers (Riasi \& Amiri Aghdaie, 2013; Riasi, 2015). The importance of leverage and volatility has been increasing in recent years as a result of the introduction of new sources of financing for the firms like funding from the shadow banking industry (Riasi \& Amiri Aghdaie, 2013; Riasi, 2015). Despite the large body of empirical literature devoted to the analysis of the leverage and volatility feedback hypotheses on asymmetric volatility, little is known about how asymmetric volatility affect the investor's asset allocation decision. The mechanisms of the leverage and volatility feedback hypotheses are different and thus may give rise to distinct effects on the optimal investment decisions for investors. For example, the leverage and volatility feedback effects would perform profound impacts on the optimal investment decisions for investors, who maximize their expected utility subject to the budget constraint made by the stochastic volatility models. In effect, the magnitude of the change in investment opportunity set is associated with the correlations between stock return and the change in volatility. The leverage and the volatility feedback effects would affect the investor's intertemporal hedging positions to hedge against the risk from the change in investment opportunity set.

This paper contributes the literature in several aspects. First, we propose a new continuous-time specification for the stock return and the change in volatility. This specification is associated with the Campbell and Hentschel's (1992) asymmetric volatility model that jointly equips with the leverage and volatility feedback effects. Specifically, our model has a flexible second moment structure, such as quadratic functional forms for the instantaneous variances of stock return and the change in volatility, the instantaneous covariance between stock return and the change in 
volatility and the time-varying state-dependent elasticity of volatility, which are all strongly associated with the optimal investment decision for investors. Next, we investigate the economic importance of the leverage and volatility feedback effects in terms of the dynamic asset allocation. Finally, the economic performances of the optimal investment decisions under various specifications and periods are calculated.

Since the seminar works of Merton $(1969,1971)$, we have known that the variation in future investment opportunities would cause investors to hedge against such risk. It in turn gives rise to an intertemporal hedging position in their optimal portfolio weights for the risky asset. In contrast to the findings in the literature, our calibrated results based on the flexible correlation specification show that the size of the intertemporal hedging demand may dominate the myopic demand and the main contribution to the intertemporal hedging demand is associated with the volatility feedback effect. It is apparent that the effects of volatility asymmetry is very critical in the dynamic asset allocation but the previous studies didn't addressed this issue. Our study would fill up this gap. Our findings are as follows. (i) the volatility feedback effect drives the intertemporal hedging demand, in contrast, the leverage effect has a minor effect on it; (ii) a longer investment horizon or a higher current volatility enhance the volatility feedback effect; (iii) ignoring the volatility feedback effect would suffer from tremendous economic loss.

\section{Volatility Asymmetries and the Model}

\subsection{The Leverage Hypothesis and the Volatility Feedback Hypotheses}

Volatility asymmetry indicates that the innovations to stock returns are negatively correlated with the innovations to stock return volatility. For example, negative (positive) returns are generally associated with upward (downward) revisions in return volatility (or conditional variance of stock return) over periods. Empirically, contemporaneous return and conditional return variance are negatively correlated. According to the financial literature, it can be explained by the leverage hypothesis or the volatility feedback hypothesis. The leverage effect proposed by Black (1976), Christie (1982) and Nelson (1991) justifies that negative shocks to returns drive up volatility. A declined stock price may deteriorate the total value of a leveraged firm. Since equity bears the full risk of the firm, it would drive up the (subsequent) volatility of equity. Thus, the hypothetical prediction of the leverage hypothesis for the cross-correlations between volatility and returns can be formalized as follows:

the leverage hypothesis: the current stock return (volatility) is negatively correlated with the current/forward volatility (current/lagged stock returns)

Alternatively, the volatility feedback hypothesis proposed by Campbell and Hentschel (1992), Bekaert and Wu (2000) and $\mathrm{Wu}$ (2001) advocated that positive shocks to volatility drive down returns. If (i) the expected stock returns increase when volatility increases and (ii) the volatility pattern is clustering and persistent, then any shock of either sign increases the expected future volatility (it is expected to persist for a period of time) and in turn raises the required returns for holding stocks, bringing down the current stock price. We can further predict that the (ex-post) forward volatility will increase and the corresponding risk will be priced in the future stock returns. Hence, the hypothetical prediction of the volatility feedback hypothesis for the cross-correlations between volatility and returns can be characterized as follows:

the volatility feedback hypothesis: the current volatility is negatively correlated with the current stock return and positively correlated with the forward volatility and stock returns.

It is apparent that although the causality of these two hypotheses are different and the mechanisms of them are independent (not mutually exclusive), their hypothetical predictions are consistent in some aspect. Moreover, both mechanisms for these two hypotheses can simultaneously contribute to volatility asymmetry, see Bekaert and Wu (2000) and $\mathrm{Wu}(2001)$.

In contrast to the above hypothetical prediction of the volatility feedback hypothesis, Bollerslev, Litvinova, and Tauchen (2006) suggested that the volatility feedback hypothesis would cause negative correlations between the returns and the lagged volatility. Their study adopted this prediction to detect the effect of the volatility feedback hypothesis on volatility asymmetry. However, this hypothetical prediction might violate one of the sufficient conditions of the volatility feedback hypothesis: the volatility risk continuously fails to be priced. The returns fail to be compensated for the corresponding upward-revised risks (or volatility).

\subsection{The Stochastic Volatility Feedback Model}

Campbell and Hentschel (1992) proposed an (discrete-time) asymmetric volatility model, in which the leverage effect and the volatility feedback effect have been involved simultaneously accounting for volatility asymmetry. 
They first assume that the unexpected changes in the dividend growth have no serial correlation at the level but a lot of them in the volatility:

$$
\begin{gathered}
\left(E_{t+1}-E_{t}\right) \sum_{j=0}^{\infty} \xi^{j} \Delta d_{t+1+j}=\sqrt{h_{t+1}} \cdot v_{t+1} \\
h_{t+1}=w+\alpha\left(\sqrt{h_{t}} \cdot v_{t}\right)^{2}+\beta h_{t}
\end{gathered}
$$

Where $v_{t+1}$ are the i.i.d Gaussian innovations with zero mean and unit variance. $h_{t+1}$ is the conditional variance of the unexpected change in the future dividend growth following an quadratic $\operatorname{GARCH}(1,1)$ process in which $\alpha$ is the ARCH parameter and $\beta$ is the GARCH parameter (Note 3 ). The parameter $\eta$ plays a particularly important role in this nonstandard GARCH model in which the $\eta$ parameter in the ARCH term $\alpha\left(\sqrt{h_{t}} v_{t}-\eta\right)^{2}$ may give rise to an asymmetric response in negative and positive shocks. If it has a positive value, the effect of a negative shock $v_{t}$ on $h_{t}$ is necessarily larger than that of a positive one. Since the magnitude of $\eta$ measure the degree of volatility asymmetry in response to the past shocks, we are able to term $\eta$ being the (conventional) leverage effect. In addition, the conditional expected return $E_{t}\left(r_{t+1}\right)$ is assumed to be a linear function of volatility of the future dividend growth:

$$
E_{t}\left(r_{t+1}\right)=\delta_{0}+\delta_{1} h_{t+1}
$$

where $\delta_{1}$ is supposed to be a positive value (it may be a negative one from empirical evidence) to reflect the fact that larger anticipated future volatility call for higher required return for holding stock.

Campbell and Hentschel (1992) proposed the following return process (Note 4):

$$
r_{t+1}=\delta_{0}+\delta_{1} h_{t+1}+(1+2 \eta \lambda) \sqrt{h_{t+1}} \cdot v_{t+1}-\lambda h_{t+1}\left(v_{t+1}^{2}-1\right),
$$

where

$$
\lambda=\frac{\delta_{1} \xi \alpha}{1-\xi(\alpha+\beta)}>0
$$

and $\xi$ is the linearization parameter (Note 5). It is important to note that the innovation $v_{t+1}$ in the future dividend growth is the only source of uncertainty and the corresponding conditional variance $h_{t+1}$ can be referred to as "fundamental volatility". The last term of $(1.5), \lambda h_{t+1}\left(v_{t+1}{ }^{2}-1\right)$, reveals a channel that the unexpected change in dividend growth, any one of either sign, gives rise to a reduction in the current return. The intuition is that: an unexpected change in the future dividend growth drives up volatility. Since volatility persists (due to GARCH effect), an investor who anticipate higher future volatility will demand larger return for hold stocks (based on the tradeoff between risk and return), which in turns causes current return to fall. Such a dynamic chain reaction in response to a return reduction has been referred to the volatility feedback effect (Campbell and Hentschel described it as no "news is good news"), and the $\lambda$ parameter appears to be a gauge on the degree of the volatility feedback effect.

By utilizing the continuous-record asymptotic theory developed by Nelson (1990, 1992), Nelson and Foster (1994) and Duan (1997) methodology, the Campbell and Hentschel's (1992) model converges to a bivariate diffusion system (the derivation is given in the Appendix B).

Proposition: If (i) the first and second moments in the discrete-time system converge to well-defined limits; (ii) the drift and diffusion terms in the diffusion limit sustain the existence and uniqueness of the solution in weak sense, then the asymmetric volatility model of Campbell and Hentschel (1992) converge to the following bivariate diffusion system:

$$
\left[\begin{array}{l}
d y_{t} \\
d h_{t}
\end{array}\right]=\left[\begin{array}{l}
\delta_{0}+\delta_{1} h_{t} \\
\psi_{0}+\psi_{1} h_{t}
\end{array}\right] d t+\left[\begin{array}{cc}
(1+2 \eta \lambda) \sqrt{h_{t}} & \sqrt{2} \lambda h_{t} \\
-2 \alpha \eta \sqrt{h_{t}} & \sqrt{2} \alpha h_{t}
\end{array}\right]\left[\begin{array}{l}
d B_{1 t} \\
d B_{2 t}
\end{array}\right],
$$

where $B_{1 t}$ and $B_{2 t}$ are two independent Brownian motions, $\psi_{0}=\left(w+\alpha \eta^{2}\right)$ and $\left.\psi_{0}=1-\alpha-\beta\right)$.

Several noticeable features are in order. First, the instantaneous variances of the stock return and the change in volatility are, respectively,

$$
\sigma_{p t}^{2}=\left[(1+2 \eta \lambda)^{2}+2 \lambda^{2} h_{t}\right] h_{t}
$$

and

$$
\sigma_{h t}^{2}=2 \alpha\left(2 \eta^{2}+h_{t}\right) h_{t},
$$

while the instantaneous covariance between stock return and the change in volatility is negative:

$$
\sigma_{p h t}^{2}=-2 \alpha\left[(1+2 \eta \lambda) \eta+\lambda h_{t}\right] h_{t}<0,
$$


All of these moments are quadratic functions of the state variable $h_{t}$. It also clearly seen that the influence of the volatility feedback effect $\lambda$ is much larger than that of the leverage effect $\eta$ as argued earlier. We therefore refer to the proposed model (6) as stochastic volatility feedback (SVF) model. Furthermore, thanks to the volatility feedback effect $\lambda$ (and to a lesser extent the leverage effect $\eta$ ), the instantaneous variance of the stock in (7) is larger, sometimes substantially larger, than the fundamental volatility $h_{t}$ corresponding to the future dividend growth and implies excessive volatility in the stock return observed by Shiller (1981) (Note 6). Also note that Bekaert and Wu (2000) and $\mathrm{Wu}$ (2001) have found some empirical evidence supporting negative relationship between stock return and the change in volatility mainly determined by the volatility feedback effect.

The elasticity of the return volatility $\sigma_{p t}$ with respect to volatility $h_{t}$ yields

$$
\frac{\mathrm{d} \ln \sigma_{p t}}{\mathrm{~d} \ln h_{t}}=\frac{1}{2}\left(1+\frac{2 \lambda^{2} h_{t}^{2}}{\sigma_{p t}^{2}}\right),
$$

which is time-varying and an increasing function with respect to $h_{t}$. It means that an upward movement in volatility $h_{t}$ is likely to drive up the elasticity of return volatility $\sigma_{p t}$, it in turn amplifies the fluctuation in stock returns and the volatility of stock return may become more volatile. This sophisticated elasticity structure is more general than that of the SV(J) model, even that of the CEV model.

\section{Dynamic Asset Allocation Analysis}

The volatility feedback effect may give rise to profound implications on the dynamic asset allocation decision of the investor. While the volatility feedback effect is kicked in, the caused change in future investment opportunities appears to be enormous in accordance with the quadratic form specifications with respect to the state variable $h_{t}$ in $\sigma_{p t}^{2}$ and $\sigma_{p h t}$. It in turn motivates a risk-averted long term investor taking adequate hedging positions to against the risk from the predictable change in investment opportunity set. We utilize a formal dynamic asset allocation model to investigate if the volatility feedback effect (as well as the leverage effect) strengthens the investor's hedging position (in magnitude) to against the intertemporal risk, and by which the total demand for stock is expected to be affected and a significant economic benefit may be created out.

\subsection{The Allocation Problem}

We utilize a standard multi-period asset allocation model, like what Liu, Longstaff, and Pan (2003) and Liu (2007) did, in which the investor equips with a power utility

$$
U(x)= \begin{cases}\frac{x^{1-\gamma}}{1-\gamma}, & \text { if } x>0, \\ -\infty, & \text { if } x \leq 0,\end{cases}
$$

where $\gamma>0$ denotes the relative risk aversion coefficient, and the second equality imposes a nonnegative condition in wealth. The nonnegative wealth constraint will rule out the arbitrage opportunities described by Harrison and Kreps (1979). Suppose that the investment opportunity set consists of a risky asset and a riskless one. The investor starts with a positive initial wealth $W_{0}>0$ and invest a fraction $\phi_{t}$ of his wealth in the risky asset at each time point $t, t \in[0, T]$ to maximize the expected utility based on his terminal wealth $W_{t}$,

$$
\max _{\left\{\phi_{t}, 0 \leq t \leq T\right\}} E_{0}\left[\frac{W_{T}^{1-\gamma}}{1-\gamma}\right] .
$$

This simplified specification facilitate to focus on the intuition behind the calibrated results, although the intermediate consumption can be easily involved.

The riskless asset is assumed to follow a deterministic process with a constant rate $\bar{r} d t$ and the risky asset is supposed to follow (6) under the economy described by the SVF model. The resulting wealth process satisfying the self- financing condition can be written by

$$
\frac{d W_{t}}{W_{t}}=\left[\phi_{t}\left(\delta_{0}+\delta_{1} h_{t}+\frac{\sigma_{p t}^{2}}{2}-\bar{r}\right)+\bar{r}\right] d t+\phi_{t}(1+2 \eta \lambda) \sqrt{h_{t}} d B_{1 t}-\phi_{t} \sqrt{2} \lambda h_{t} d B_{2 t},
$$

where $\sigma_{p t}^{2} / 2$ is the adjustment term from Ito formula.

In order to solve the optimal portfolio weight $\phi_{t}$, we utilize the standard stochastic control approach. Following Merton (1974) and Liu (2007), while the indirect utility function $J$ is defined by

$$
J\left(W_{t}, h_{t}, t\right)=\max _{\left\{\phi_{t}, 0 \leq t \leq T\right\}} E_{t}\left[U\left(W_{t}\right)\right],
$$

the principle of stochastic control results in the following Hamilton-Jacobi-Bellman (HJB) equation: 


$$
0=\sup _{\phi_{t}}\left\{j+J_{W} W_{t}\left[\phi_{t}\left(\delta_{0}+\delta_{1} h_{t}+\frac{\sigma_{p t}^{2}}{2}\right)+\bar{r}\right]+\frac{J_{W W}}{2} W_{t}^{2} \phi_{t}^{2} \sigma_{p t}^{2}+\left(\psi_{0}-\psi_{1} h_{t}\right) J_{h}+\frac{J_{h h}}{2} \sigma_{h t}^{2}-J_{W h} \phi_{t} \sigma_{p h t}\right\},
$$

where $j, J_{W}, J_{h}$ denote the first-order derivatives of $J$ with respect to $t, W_{t}, h_{t}$ and $J_{W W}, J_{h h}, J_{W h}$ obviously denote the corresponding second-order derivatives.

We then conjecture the functional form of the indirect utility function $J$ being

$$
J\left(W_{t}, h_{t}, t\right)=\frac{w_{t}^{1-\gamma}}{1-\gamma} A\left(h_{t}, t\right)
$$

Based on the conjectured form, we take first- and second-order derivatives of $J$ with respect to $W_{t}, h_{t}, t$ in place of the corresponding terms in HJB equation (13). After that, we take the first-order derivative of HJB equation with respect to $\phi_{t}$ by achieving the optimal solution so that the optimal portfolio weight, given the conjectured function, yields

$$
\phi_{t}^{*}=\frac{1}{\gamma}\left(\frac{\delta_{0}+\delta_{1} h_{t}-\bar{r}+\frac{\sigma_{p t}^{2}}{2}}{\sigma_{p t}^{2}}\right)-\frac{A_{h}}{\gamma A}\left(\frac{\sigma_{p h t}}{\sigma_{p t}^{2}}\right)
$$

The value function $A$ satisfies the following partial differential equation

$$
0=\frac{\dot{A}}{1-\gamma}+\left[\phi_{t}\left(\delta_{0}+\delta_{1} h_{t}+\frac{\sigma_{p t}^{2}}{2}-\bar{r}\right)+\bar{r}-\frac{\gamma}{2} \phi_{t}^{* 2} \sigma_{p t}^{2}\right]+\left(\psi_{0}-\psi_{1} h_{t}\right) \frac{A_{h}}{1-\gamma}+\frac{A_{h h}}{2(1-\gamma)} \sigma_{h t}^{2}-A_{h} \phi_{t}^{*} \sigma_{p h t},
$$

where $\dot{A}=\partial A / \partial t, A_{h}=\partial A / \partial h$ and $A_{h h}=\partial^{2} A / \partial^{2} h$.

The optimal portfolio weight (15) consists of two components: the first one is called the myopic demand and the second one is called the intertemporal hedging demand. The myopic demand is obviously irrelevant to the value function $A$ but depends on the reciprocal of $\gamma$ and the return's mean-variance ratio, which has been viewed as the current price of risk on stock market. The influence of the volatility feedback effect on the myopic demand goes through the stock return variance $\sigma_{p t}^{2}$. Since the mean-variance ratio reflects only current market condition, the demand based on this ratio turns to be myopic. The intertemporal hedging demand depends on the reciprocal of $\gamma$, the covariance between stock return and the change in state variance $\sigma_{p h t}$, the stock return variance $\sigma_{p t}^{2}$ and the curvature of the value function $A_{h} / A$. The change in volatility is supposed to induce a change in future investment opportunities so that the invested wealth is necessarily affected. As mentioned in the previous sections, the magnitude of the change in investment opportunity set depends on the specifications of $\sigma_{p t}^{2}$ and $\sigma_{p h t}$, which are heavily affected by the volatility feedback effect. The curvature of the value function $A$ reflects the response of the investor against the change in investment opportunity set caused by the change in state variable $h_{t}$ via $\sigma_{p t}^{2}$ and $\sigma_{\text {pht }}$.

\subsection{Optimal Portfolio Weights and Horizon Effect}

Quantitative analysis for the optimal portfolio weight under the economy described by the SVF model mainly pertains to the solving of the partial differential equation (16), calibrating the value function $A$. The whole calibration for the optimal portfolio weight is done under the following setups: the relative risk coefficient $\gamma$ is set to be 3, and the investment horizon $T$ is 6 months for a short-term investment and 120 months for a long-term investment (Note 7). The optimal portfolio weight is calibrated with respect to the state variable $h_{t}$. 

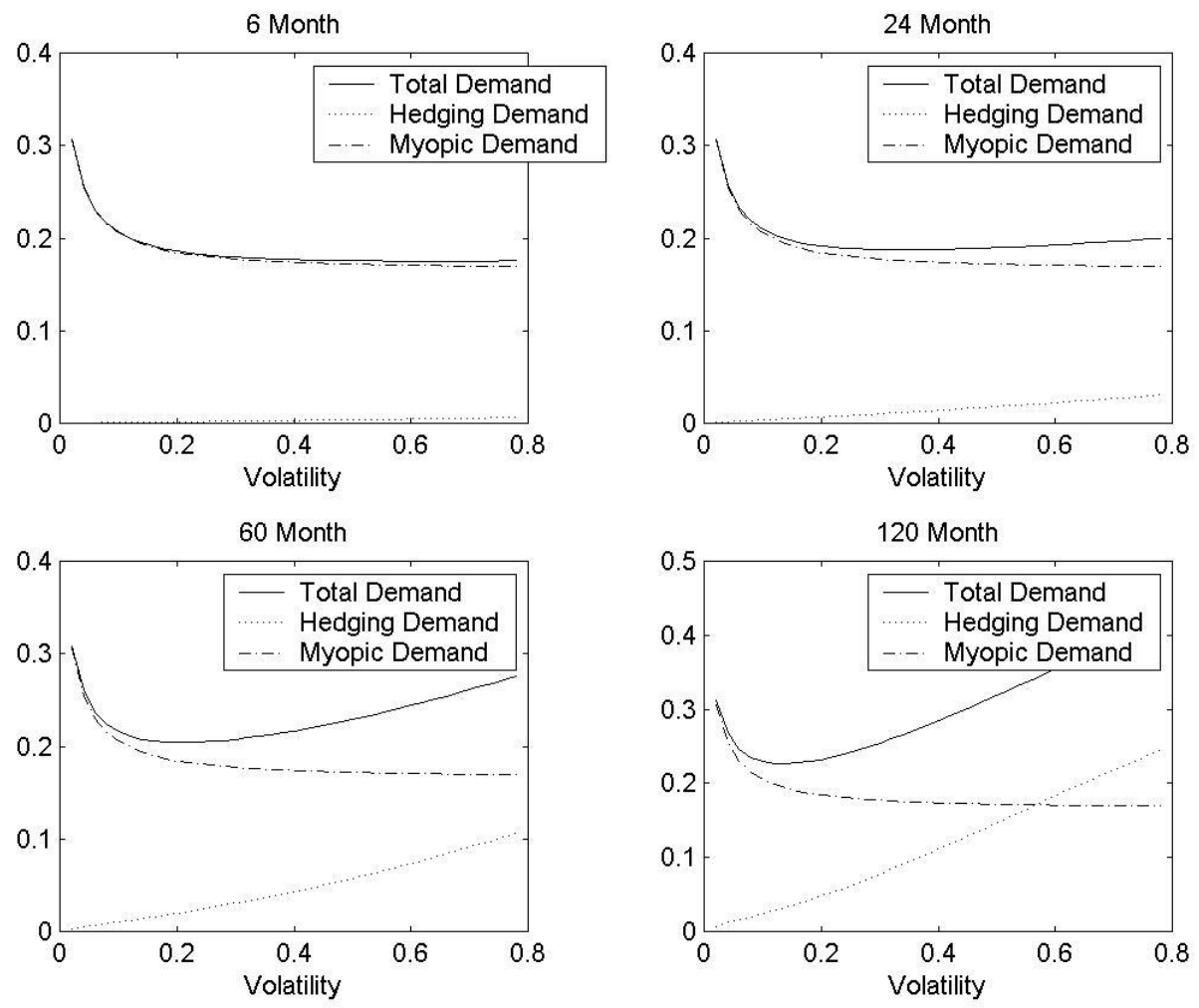

Figure 1. The Investment Horizon and Optimal Portfolio Weights

Figure 1 plots the proportion of wealth invested in stock return under different investment horizons. The investment horizons are divided by six months, two years, five years and ten years. When the investment horizon is six months, the total demand is very closed to myopic demand, the intertemporal hedging demand approaches to zero. At the five-year investment horizon, the total demand exhibits a $\mathrm{U}$ sharp feature, and the intertemporal hedging demand can dominate the the myopic demand when the investment horizon be ten years. The interpretations of these results can be decomposed by two aspects. First, according to the predictability on stock return, high volatility implies good investment opportunities in the future because it will be compensated in risk premium. Besides, the longer is investment horizon, the more possibility for the volatility reverts to its long-term return, implying lower risk burden of holding stock over long-term horizon. Second, the volatility feedback effect be an amplification mechanism making the shifts of investment opportunities being persist. A longer investment horizon, the investors more concern about the persistence shifts on investment opportunities. They have strong motivation to hedging these intertemporal risks. Here, the amplification mechanism via volatility feedback effect means that a piece of bad news drops down the stock price, the debt-equity ratio increase makes the volatility increase. Then this increase volatility drops down stock price again because the required return on stock increase. The re-drop stock price increases the volatility again. This recursive procedure keeps the movements on future investment opportunities. 


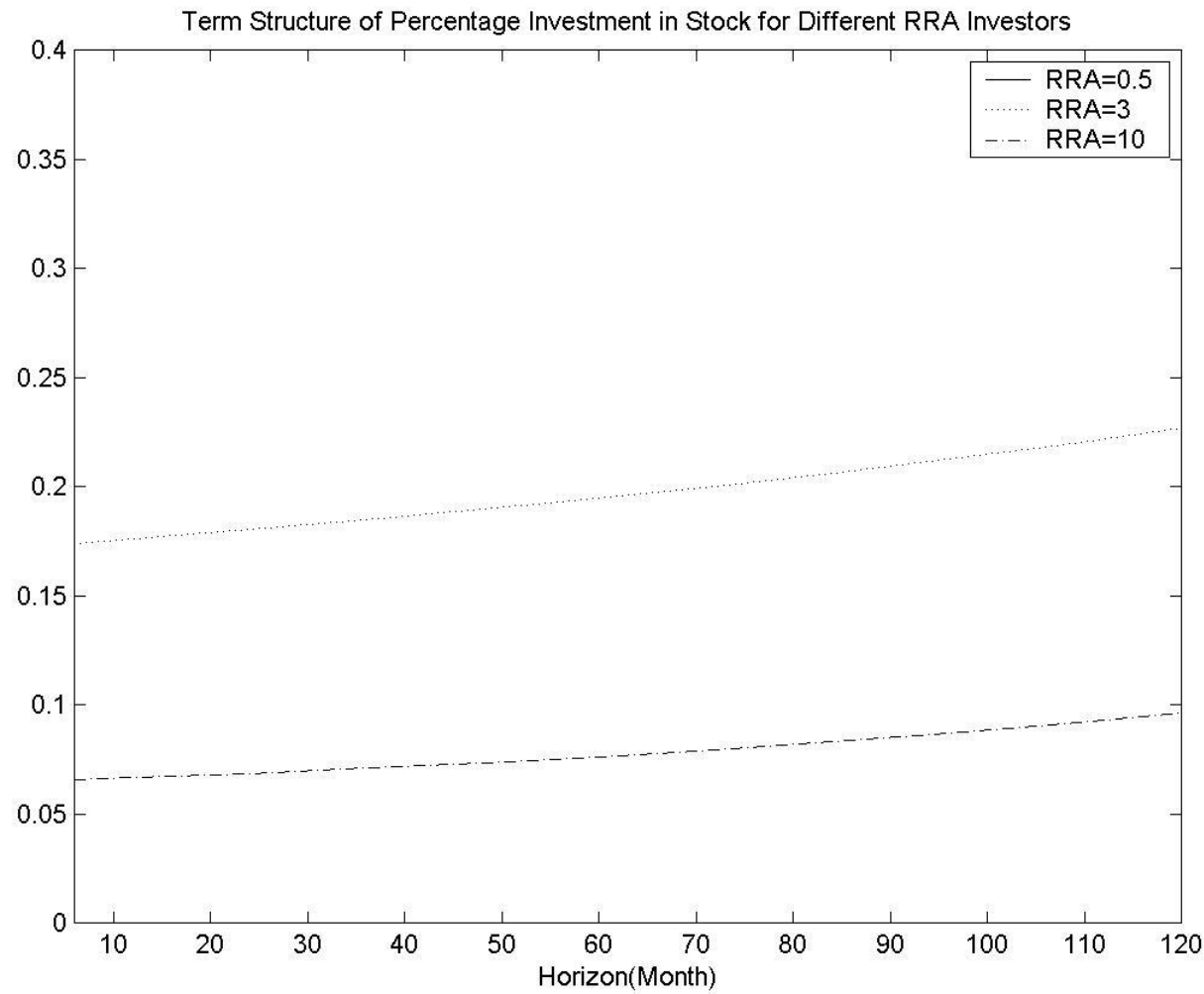

Figure 2. Horizon Effects and Risk Aversion

Figure 2 plots the term structure of optimal portfolio weights under different $\gamma$. As investment horizon increase, the conservative investors $(\gamma=3,5)$ increase the proportion of wealth invested in stock. Whereas, the aggressive investors $(\gamma=0.5)$ decrease the proportion of wealth invested in stock. Because the aggressive investors have negative intertemporal hedging demand. Besides, the magnitude of percentage investment in stock also depends on $\gamma$. The investors with higher $\gamma$ hold less stock than the investors with lower $\gamma$. In particular, the aggressive investors may have a leveraged portfolio strategy because their percentage investment in stock allows to greater than one.

\subsection{The Economic Benefits and the Volatility Feedback Effect}

It is known that the volatility feedback effect given a proper investment horizon may give rise to profound influence on the stock demand of the investor based on the previous calibration results. In this section, we seek to assess the economic benefit created by the volatility feedback effect.

Another formal metric assessing a given optimal portfolio weight advocated by the financial literature is the utility-based benefit and this is the most relevant metric as it quantifies benefits based on investor preference. Certainty equivalent wealth (CEW) is a metric to acknowledge what will be lost or gained if we ignore/involve some sorts of risk sources. Following Brennan, Schwartz and Lagnado (1998) and Xia (2001), we are able to compare different optimal portfolio weights directly because CEW is doing the economic value for a given dynamic trading strategy. The value of CEW for a given optimal portfolio weight is the amount of money that makes the investor indifferent between receiving certainty CEW at the time $T$ and investing $\$ 1$ today up to the time $T$ utilizing the given strategy. In other word, CEW is the monetary value of a given optimal portfolio weight inside an investor and then has been defined by

or

$$
\frac{C E W^{1-\gamma}\left(\phi^{*}\right)}{1-\gamma}=J\left(\$ 1, h_{t}, t\right)=A\left(h_{t}, t ; \phi_{t}^{*}\right) \frac{\$ 1^{1-\gamma}}{1-\gamma} .
$$

$$
\operatorname{CEW}\left(\phi^{*}\right)=A\left(h_{t}, t ; \phi_{t}^{*}\right)^{\frac{1}{1-\gamma}} .
$$

The main results of the $C E W$ values over various cases of the SVF model and four different sample periods are also reported. 


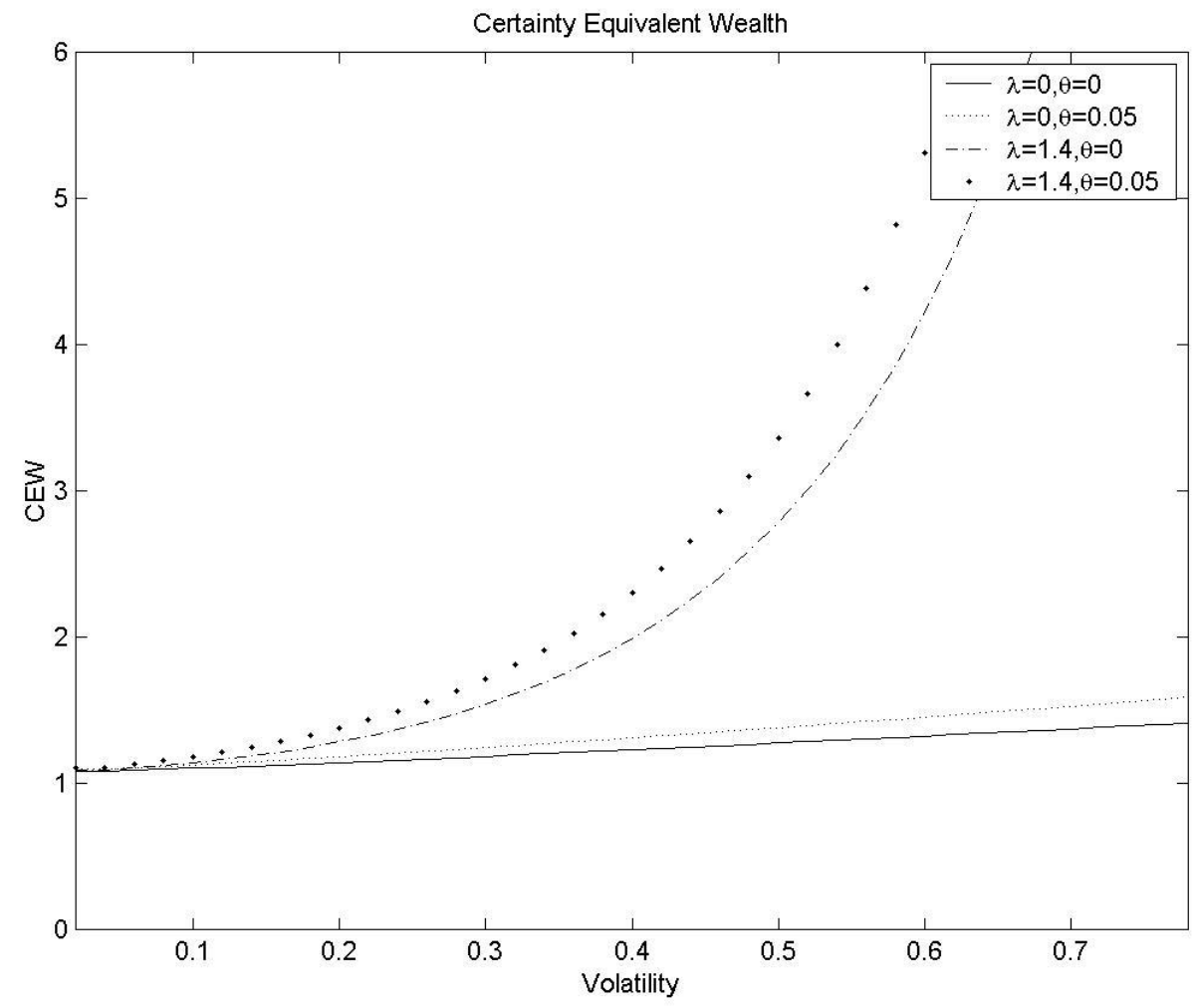

Figure 3. Certainty Equivalent Wealth and Asymmetric Volatility

To access the economic importance of feedback mechanism, we run a horse race among four strategies: no asymmetric volatility $(\lambda=\eta=0)$, only leverage effect $(\lambda=0, \eta=0.05)$, only volatility feedback effect $(\eta=0, \lambda=1.4)$ and both effect $(\lambda=1.4, \eta=0.05)$. Figure 3 exhibits the $C E W$ s of these four strategies. Here, we set the relative risk aversion coefficient to be three and fix the investment horizon to be ten years. The $C E W \mathrm{~s}$ of strategies that consider volatility feedback effect are obviously superior than the $C E W$ s of strategies that ignoring volatility feedback effect. The gap of $C E W$ between these strategies becomes large as the volatility increase. Whether consider the volatility feedback effect of not on the optimization decision significantly affect the valuation of the strategy. 


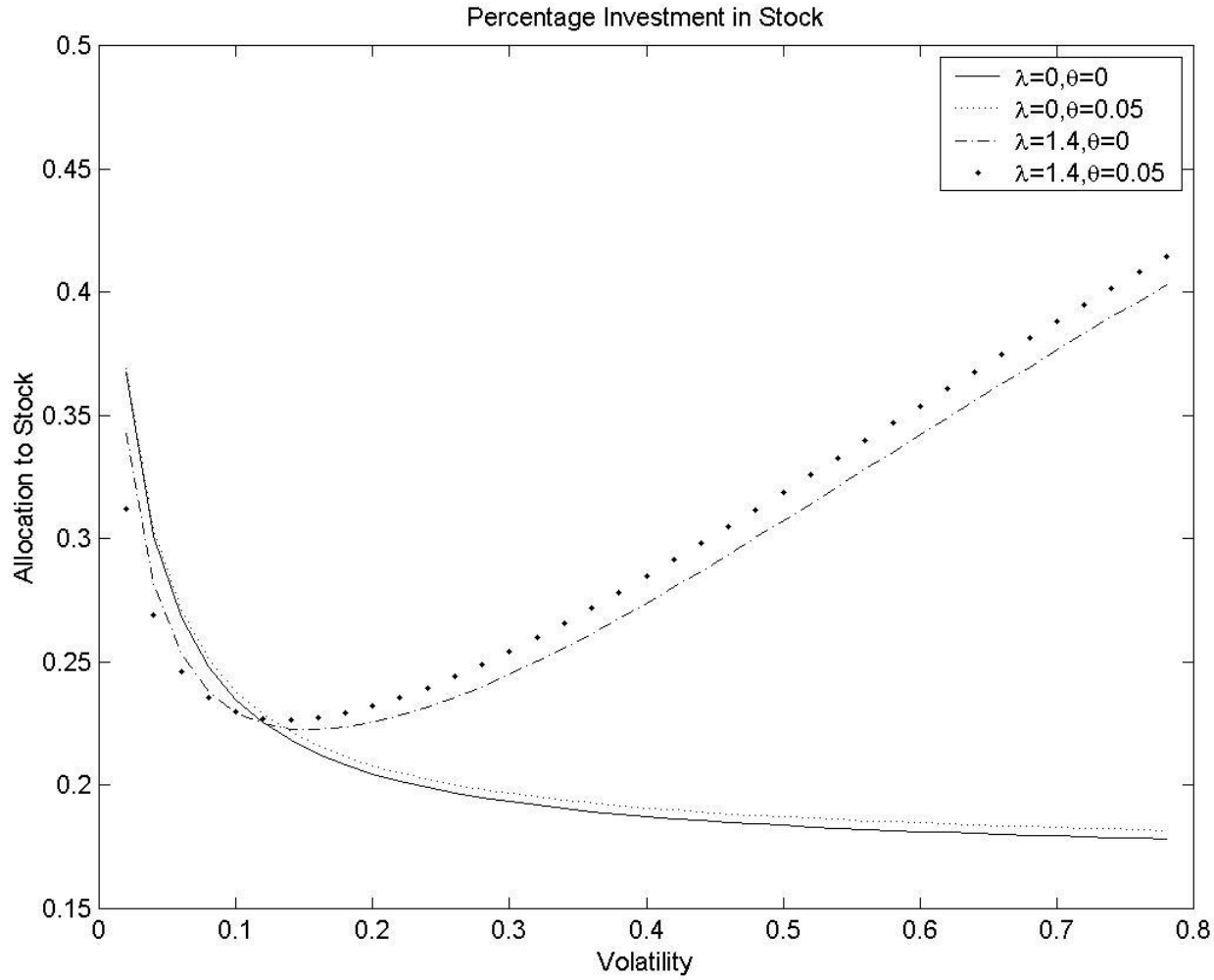

Figure 4. Optimal Portfolio Weights and Asymmetric Volatility

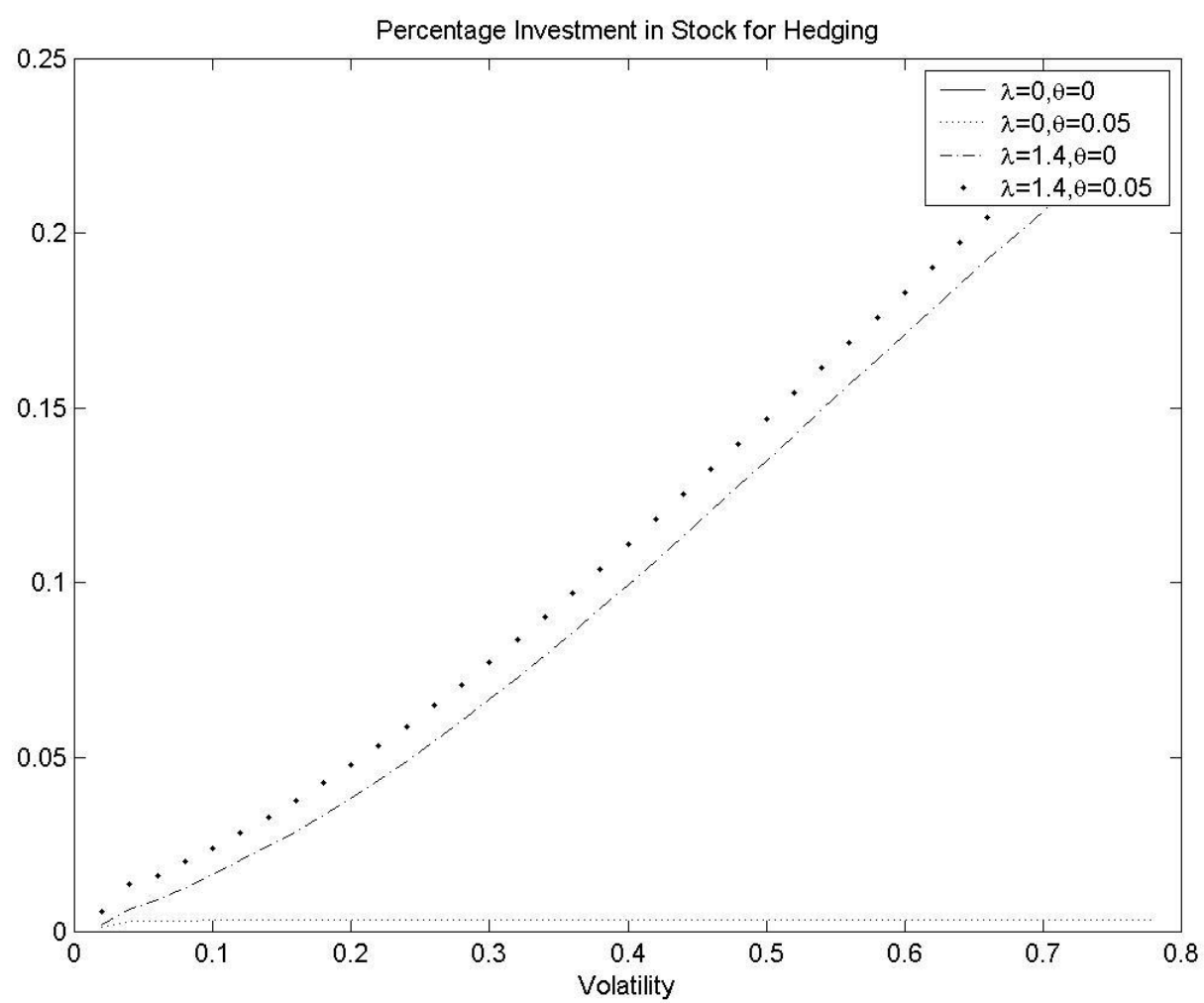

Figure 5. Intertemporal Hedging and Asymmetric Volatility 
The interpretations of $C E W^{\prime}$ 's results are based on the following two features. Figure 4 exhibits the percentage investment in stock among these four strategies and Figure 5 plots the proportion of wealth invested in stock for hedging among these four strategies. The $\gamma$ is three and investment horizon is ten years in these two features. In Figure 3 , the strategies that consider the volatility feedback effect exhibit $U$ sharp feature, however, the strategies ignore the volatility feedback effect exhibit monotonic decreasing feature. In Figure 5, the intertemporal hedging demand for the strategies with volatility feedback effect are much more than the strategies without volatility feedback effect. In particular, the only leverage effect strategy has no significant intertemporal hedging motivation even though the leverage effect allows asymmetric volatility. Without the amplification mechanism from volatility feedback, only leverage effect cannot generate enough movements on investment opportunities to induce strong intertemporal hedging motivation. This explanation is very similar to that of Liu (2001), on other hand, it sheds light on the importance of volatility feedback effect.

The high $C E W$ on the strategies with volatility feedback effect is coming from the benefit of "time diversification" or horizon effect. These strategies diversify the risk burden of holding stock over the long period of time. For example, although today's volatility is high (the price is low), future return will compensate this risk in risk premium and the risk (volatility) may diminish in the future due to the mean-reverting property. As a result, a conservative long-term investor would like to hold much more stock for the long-term benefits under this situation.

\section{Conclusion}

The volatility feedback effect and the leverage effect can be attributed to the two major mechanisms to cause the asymmetric responses for the positive and negative shocks in stock return and return volatility. This paper devises a stochastic volatility feedback (SVF) model to investigate the economic importance of the leverage and volatility feedback effects. We perform the dynamic asset allocation model under various settings for the budget constraint and then compare the economic performances for the corresponding optimal investment strategies. Our findings are as follows. (i) the volatility feedback effect drives the intertemporal hedging demand, in contrast, the leverage effect has a minor effect on it; (ii) a longer investment horizon or a higher current volatility enhance the volatility feedback effect; (iii) ignoring the volatility feedback effect would suffer from tremendous economic loss.

This paper contributes the literature in several aspects. First, we propose a new continuous-time specification for the stock return and the change in volatility. Next, we investigate the the economic importance of the leverage and volatility feedback effects in terms of the dynamic asset allocation. Finally, the economic performances of the optimal investment decisions under various specifications and periods are calculated.

\section{References}

Andersen, T. G., \& Lund, J. (1997). Estimating continuous-time stochastic volatility models of the short-term interest rate. Journal of econometrics, 77(2), 343-377. http://dx.doi.org/10.1016/S0304-4076(96)01819-2

Andersen, T. G., Benzoni, L., \& Lund, J. (2002). An empirical investigation of continuous-time equity return models. The Journal of Finance, 57(3), 1239-1284. http://dx.doi.org/10.1111/1540-6261.00460

Bekaert, G., \& Wu, G. (2000). Asymmetric volatility and risk in equity markets. Review of Financial Studies, 13(1), 1-42. http://dx.doi.org/ 10.1093/rfs/13.1.1

Black, F. (1976). \{Stuedies of stock price volatility changes\}. http://www.citeulike.org/group/2384/article/7656400

Bollerslev, T., Litvinova, J., \& Tauchen, G. (2006). Leverage and volatility feedback effects in high-frequency data. Journal of Financial Econometrics, 4(3), 353-384. http://dx.doi.org/10.1093/jjfinec/nbj014

Brennan, M. J. (1998). The role of learning in dynamic portfolio decisions. European finance review, 1(3), 295-306. http://dx.doi.org/10.1023/A:1009725805128

Brennan, M. J., Schwartz, E. S., \& Lagnado, R. (1997). Strategic asset allocation. Journal of Economic dynamics and Control, 21(8), 1377-1403. http://dx.doi.org/10.1016/S0165-1889(97)00031-6

Campbell, J. Y., Chacko, G., Rodriguez, J., \& Viceira, L. M. (2004). Strategic asset allocation in a continuous-time VAR model. Journal of Economic Dynamics and Control, 28(11), 2195-2214. http://dx.doi.org/10.1016/j.jedc.2003.09.005

Campbell, J. Y., \& Hentschel, L. (1992). No news is good news: An asymmetric model of changing volatility in stock returns. Journal of financial Economics, 31(3), 281-318. http://dx.doi.org/10.1016/0304-405X(92)90037-X

Campbell, J. Y., Lo, A. W. C., \& MacKinlay, A. C. (1997). The econometrics of financial markets (Vol. 2, pp. 149-180). Princeton, NJ: princeton University press. 
http://economicpolicy.oxfordjournals.org/content/economicpolicy/11/23/local/back-matter.pdf

Campbell, J. Y., \& Viceira, L. M. (1996). Consumption and portfolio decisions when expected returns are time varying (No. w5857). National Bureau of Economic Research. http://dx.doi.org/10.3386/w5857

Campbell, J. Y., \& Viceira, L. M. (2002). Strategic asset allocation: portfolio choice for long-term investors. Oxford University Press, USA. https://goo.gl/rTqgu6

Chacko, G., \& Viceira, L. M. (2005). Dynamic consumption and portfolio choice with stochastic volatility in incomplete markets. Review of Financial Studies, 18(4), 1369-1402. http://dx.doi.org/10.1093/rfs/hhi035

Chernov, M., Gallant, A. R., Ghysels, E., \& Tauchen, G. (2003). Alternative models for stock price dynamics. Journal of Econometrics, 116(1), 225-257. http://dx.doi.org/10.1016/S0304-4076(03)00108-8

Christie, A. A. (1982). The stochastic behavior of common stock variances: Value, leverage and interest rate effects. Journal of financial Economics, 10(4), 407-432. http://dx.doi.org/10.1016/0304-405X(82)90018-6

Duan, J. C. (1997). Augmented GARCH (p, q) process and its diffusion limit.Journal of Econometrics, 79(1), 97-127. http://dx.doi.org/10.1016/S0304-4076(97)00009-2

French, K. R., Schwert, G. W., \& Stambaugh, R. F. (1987). Expected stock returns and volatility. Journal of financial Economics, 19(1), 3-29. http://dx.doi.org/10.1016/0304-405X(87)90026-2

Heston, S. L. (1993). A closed-form solution for options with stochastic volatility with applications to bond and currency options. Review of financial studies, 6(2), 327-343. http://dx.doi.org/10.1093/rfs/6.2.327

Johannes, M. S., Polson, N., \& Stroud, J. R. (2002). Sequential optimal portfolio performance: Market and volatility timing. Available at SSRN 304976. http://dx.doi.org/10.2139/ssrn.304976

Liu, J. (2001). Dynamic portfolio choice and risk aversion. Preprint, Anderson School of Management, UCLA.

Liu, J. (2007). Portfolio selection in stochastic environments. Review of Financial Studies, 20(1), 1-39. http://dx.doi.org/10.1093/rfs/hhl001

Liu, J., Longstaff, F. A., \& Pan, J. (2003). Dynamic asset allocation with event risk. The Journal of Finance, 58(1), 231-259. http://dx.doi.org/10.1111/1540-6261.00523

Nelson, D. B. (1990). ARCH models as diffusion approximations. Journal of econometrics, 45(1), 7-38. http://dx.doi.org/10.1016/0304-4076(90)90092-8

Nelson, D. B. (1991). Conditional heteroskedasticity in asset returns: A new approach. Econometrica: Journal of the Econometric Society, 347-370. http://dx.doi.org/10.2307/2938260

Nelson, D. B. (1994). Asymptotic filtering theory for multivariate ARCH models. http://dx.doi.org/ 10.3386/t0162

Riasi, A., \& Amiri Aghdaie, S. F. (2013). Effects of a Hypothetical Iranian Accession to the World Trade Organization on Iran's Flower Industry. Consilience: The Journal of Sustainable Development, 10(1), 99-110. http://dx.doi.org/10.7916/D8HQ3ZK8

Riasi, A. (2015). Competitive Advantages of Shadow Banking Industry: An Analysis Using Porter Diamond Model. Business Management and Strategy, 6(2), 15-27. http://dx.doi.org/10.5296/bms.v6i2.8334

Wu, G. (2001). The determinants of asymmetric volatility. Review of Financial Studies, 14(3), 837-859. http://dx.doi.org/10.1093/rfs/14.3.837

Xia, Y. (2001). Learning about predictability: The effects of parameter uncertainty on dynamic asset allocation. The Journal of Finance, 56(1), 205-246. http://dx.doi.org/ 10.1111/0022-1082.00323 


\section{Notes}

Note 1. Campbell and Hentschel (1992) pointed out that five largest one-day movement in S\\&P 500 index since World War II, four out of five are dropping in the index, of the ten largest movements, eight out of ten are dropping.

Note 2. Campbell, Lo and MacKinlay (1997) adopted these two terms explaining volatility asymmetry.

Note 3. Their sum of $\alpha+\beta$ must be smaller than one but is usually quite close to one, reflecting the degree of persistence in $h_{t+1}$.

Note 4. By repeated substitution using (1.7), the unexpected change in the future return, i.e. the second term of (1.4), can be expressed by:

$$
\left(E_{t+1}-E_{t}\right) \sum_{j=1}^{\infty} \xi^{j} r_{t+1+j}=-2 \eta \lambda \sqrt{h_{t+1}} \cdot v_{t+1}+\lambda h_{t+1}\left(v_{t+1}^{2}-1\right),
$$

where the $\lambda$ parameter is expressed in (1.9). Combining the above term and (1.5), (1.8) is straightforwardly available.

Note 5. The whole derivation can be found in Campbell and Shiller (1998) and Campbell (1991). $\xi$, in fact, is the average ratio of the stock price to the sum of the price and the dividend and in (1.4) we have imposed the terminal condition: $\lim _{j} \rightarrow \infty \xi^{j} r_{t+j}=0$

Note 6. It should be clear by now that the state variable $h_{t}$ in the SV and SVJ model is the stock return volatility. In contrast, the state variable $h_{t}$ in the SVF model is the conditional variance of unexpected change in the future dividend growth as defined in (1.2)

Note 7. The detail procedure of model calibration is addressed in appendix A. 